\title{
RESEARCH HIGHLIGHT Metabotropic glutamate receptor 5 signaling and appetitive Pavlovian behavior: implications for the treatment of addiction
}

\author{
Jocelyn M. Richard (10 ${ }^{1}$ \\ Neuropsychopharmacology (2019) 44:1516-1517; https://doi.org/10.1038/s41386-019-0375-y
}

\begin{abstract}
A key challenge in the treatment of drug addiction is that people suffering from addiction are at continued risk of relapsing even after long periods of successful abstinence. The persistent risk of relapse is a central part of what makes addiction one of the most deadly and devastating public health challenges. The gravity of this existing public health crisis has only been intensified by the emergence of the opioid epidemic. In many cases, relapse is elicited by environmental cues and contexts that were previously associated with drug availability or drug use. These cues can evoke intense cravings, which may lead people to seek out drugs despite a stated desire to abstain. In order to develop successful long-term treatments for addiction, we need to address the biological mechanisms by which cues evoke craving, long after patients have stopped taking drugs of abuse.
\end{abstract}

Metabotropic glutamate receptors (mGluRs) have shown promise as a potential target for the treatment of substance abuse and the reduction of relapse rates. Drugs acting on receptors in this family are reported to modulate drug-seeking behaviors in preclinical models of drug self-administration and relapse-like behavior [1]. Specifically, negative allosteric modulators of type 5 mGluRs have been shown to attenuate to drugseeking behavior in multiple models of relapse [2, 3]. These models include reinstatement of drug-seeking behavior that is elicited or reinforced by drug-associated cues. Fenobam, a drug that was recently revealed to act an mGluR5 negative allosteric modulator [4], showed promise in clinical testing for the treatment of anxiety in the early 1980s. Unfortunately, concerns have been raised about the impact of these types of drugs on learning and memory [5] and whether these off-target effects actually underly their efficacy in reducing cue-induced reinstatement behaviors. A major open question regarding the role of mGluR5s is the behavioral mechanism by which they contribute to relapse elicited by drug-associated cues. Do these receptors contribute to the expression of Pavlovian-conditioned behaviors more generally? And if so, is it possible to disentangle the role of mGluR5 signaling in relapse-related behavior from the contributions of this signaling to adaptive responses to Pavlovian reward cues? Answers to these questions are critical for the future refinement of pharmacotherapies targeting these receptors for the treatment of addiction.

In their recent Neuropsychopharmacology article, Khoo and colleagues [6] reported that mGluR5 signaling exerts distinct effects on context discrimination and cue-elicited behavior, depending on the brain site of action. The authors examined the impact of a negative allosteric modulator of mGluR5, 3-((2methyl-4-thiazolyl)ethynyl)pyridine (MTEP), on cue-elicited behavior after Pavlovian conditioning with an auditory cue that predicted delivery of a fructose-glucose solution. Rats were tested in both a reward-associated context, in which the cue had previously predicted reward delivery, and in a neutral context, in which rats had experienced an alternative cue that predicted no reward. Systemic injections of MTEP reduced the ability of the reward-associated cue to elicit entry to the reward port, regardless of the context in which rats were tested. This result suggests that reductions in cue-elicited relapse-like behavior following MTEP could be related to more general disruptions in behavioral responses to Pavlovian cues. If so, then brain-wide modulation of mGluR5 signaling may have unintended consequences in the form of reductions in adaptive cue-driven behavior. Importantly, the authors next set out to determine whether mGluR5 signaling impacts Pavlovian responses at the same sites where it has been shown to impact cue-induced reinstatement.

Khoo et al. examined the impact of local infusion of MTEP in either the nucleus accumbens (NAc) or basolateral amygdala (BLA), two areas of the brain that have been shown to mediate mGluR5 effects in models of relapse to ethanol- or cocaine-seeking $[2,3]$, and to mediate cue-driven behavior more generally $[7,8]$. Intriguingly, local injection of MTEP in either site did not recapitulate the effect pattern observed after systemic negative allosteric modulation of mGluR5. Instead, NAc MTEP had no discernable effect on cue-elicited port entry behavior, and the impact of BLA MTEP depended both on the context in which rats were tested and the anteroposterior location of the injection site. When Khoo et al. first assessed the impact of MTEP infusion in BLA, they saw no overall effect. But, when they analyzed the topography of post-MTEP changes in behavior, their analysis suggested that MTEP at more anterior BLA sites might suppress cue-elicited port entry behavior across both contexts, much like the impact of systemic MTEP. Instead, a follow-up experiment revealed that anterior BLA MTEP potentiated the impact of context. That is, when rats were tested in the reward-associated context, MTEP increased their port entry behavior during the cue, but when rats were tested in the neutral context, MTEP had the opposite effect. Rather than disrupting Pavlovian cue-driven behavior, anterior BLA MTEP appeared to make rats better at using contextual cues to modulate this Pavlovian cue-driven behavior.

Overall, the authors demonstrate that mGluR5 signaling contributes to reward-related behaviors elicited by Pavlovian cues, but that these systemic effects are not mediated by mGluR5 actions in the BLA or NAc. This may seem surprising, given the previously reported impact of BLA or NAC MTEP on cue-induced reinstatement behavior. Yet, reward-associated cues can drive a variety of distinct behaviors via dissociable neural (and

${ }^{1}$ Department of Neuroscience, University of Minnesota, Minneapolis, MN 55455, USA

Correspondence: Jocelyn M. Richard (richardj@umn.edu)

Received: 11 March 2019 Accepted: 18 March 2019

Published online: 9 April 2019 
psychological) mechanisms. Effectively treating addiction requires that we are able to disentangle maladaptive cue-related behaviors from those that are required for normal day-to-day functioning. For instance, cue-induced reinstatement of drug seeking requires that animals perform a previously trained operant response for contingent presentations of a previously learned drug cue. Cues that are able to reinforce responding in this type of test are said to have incentive motivational value. This property of cues, which requires mGluR5 signaling during acquisition [9], is biologically dissociable from the ability of cues to provide predictive information about upcoming reward delivery and to elicit approach to the location of that reward delivery. It remains to be seen whether mGluR5 signaling in any one brain region is required for cue-driven port entry behavior. If mGluR5 signaling effects on distinct cue-driven behaviors can be mapped onto dissociable neural substrates, then this information can be used to more selectively target mGluR5-based strategies for the treatment of addiction or other disorders involving maladaptive responses to reward-associated cues.

\section{FUNDING AND DISCLOSURE}

J.M.R. is supported by an NIH/NIAAA grant R00AA025384. The author declares no competing interests.

\section{ADDITIONAL INFORMATION}

Publisher's note: Springer Nature remains neutral with regard to jurisdictional claims in published maps and institutional affiliations.

\section{REFERENCES}

1. Caprioli D, Justinova Z, Venniro M, Shaham Y. Effect of novel allosteric modulators of metabotropic glutamate receptors on drug self-administration and relapse: a review of preclinical studies and their clinical implications. Biol Psychiatry. 2018;84:180-92.

2. Backstrom P, Bachteler D, Koch S, Hyytia P, Spanagel R. mGluR5 antagonist MPEP reduces ethanol-seeking and relapse behavior. Neuropsychopharmacology. 2004:29:921-8.

3. Knackstedt LA, Trantham-Davidson HL, Schwendt M. The role of ventral and dorsal striatum mGluR5 in relapse to cocaine-seeking and extinction learning. Addict Biol. 2014;19:87-101.

4. Porter RHP, Jaeschke G, Spooren W, Ballard TM, Buttelmann B, Kolczewski S, et al Fenobam: a clinically validated nonbenzodiazepine anxiolytic is a potent, selective, and noncompetitive mGlu5 receptor antagonist with inverse agonist activity. J Pharmacol Exp Ther. 2005;315:711-21.

5. Jacob W, Gravius A, Pietraszek M, Nagel J, Belozertseva I, Shekunova E, et al. The anxiolytic and analgesic properties of fenobam, a potent mGlu5 receptor antagonist, in relation to the impairment of learning. Neuropharmacology. 2009;57:97-108.

6. Khoo SY-S, LeCocq MR, Deyab GE, Chaudhri N. Context and topography determine the role of basolateral amygdala metabotropic glutamate receptor 5 in appetitive Pavlovian responding. Neuropsychopharmacology. 2019. https://doi.org/10.1038/ s41386-019-0335-6.

7. Chaudhri N, Sahuque LL, Schairer WW, Janak PH. Separable roles of the nucleus accumbens core and shell in context- and cue-induced alcohol-seeking. Neuropsychopharmacology. 2010;35:783-91.

8. Sciascia JM, Reese RM, Janak PH, Chaudhri N. Alcohol-seeking triggered by discrete pavlovian cues is invigorated by alcohol contexts and mediated by glutamate signaling in the basolateral amygdala. Neuropsychopharmacology. 2015;40: 2801-12.

9. O'Connor EC, Crombag HS, Mead AN, Stephens DN. The mGluR5 antagonist MTEP dissociates the acquisition of predictive and incentive motivational properties of reward-paired stimuli in mice. Neuropsychopharmacology. 2010;35: 1807-17. 